\title{
Bayesian Joint Detection-Estimation of cerebral vasoreactivity from ASL fMRI data
}

\author{
Thomas Vincent $^{1}{ }^{\star}$, Jan Warnking ${ }^{2}$, Marjorie Villien ${ }^{2}$, Alexandre Krainik² \\ Philippe Ciuciu ${ }^{3}$, and Florence Forbes ${ }^{1}$ \\ (1) INRIA, MISTIS, Grenoble University, LJK, Grenoble, France \\ (2) INSERM U836-UJF-CEA-CHU (GIN), Grenoble, France \\ ${ }^{(3)} \mathrm{CEA} / \mathrm{DSV} / \mathrm{I}^{2} \mathrm{BM}$ NeuroSpin center, Bât. 145, F-91191 Gif-sur-Yvette, France
}

\begin{abstract}
Although the study of cerebral vasoreactivity using fMRI is mainly conducted through the BOLD fMRI modality, owing to its relatively high signal-to-noise ratio (SNR), ASL fMRI provides a more interpretable measure of cerebral vasoreactivity than BOLD fMRI. Still, ASL suffers from a low SNR and is hampered by a large amount of physiological noise. The current contribution aims at improving the recovery of the vasoreactive component from the ASL signal. To this end, a Bayesian hierarchical model is proposed, enabling the recovery of perfusion levels as well as fitting their dynamics. On a single-subject ASL real data set involving perfusion changes induced by hypercapnia, the approach is compared with a classical GLM-based analysis. A better goodness-of-fit is achieved, especially in the transitions between baseline and hypercapnia periods. Also, perfusion levels are recovered with higher sensitivity and show a better contrast between gray- and white matter.
\end{abstract}

Key words: fMRI, ASL, cerebral vasoreactivity, deconvolution, Bayesian analysis, Monte Carlo Markov Chain inference.

\section{Introduction}

Cerebral vasoreactivity (CVR) is a fundamental function of brain perfusion, involved in the dynamic regulation of blood flow as a function of metabolic demand. Vasomotor impairments are known or hypothesized in a variety of diseases $[1,2]$ and reliable measurement of CVR may be a valuable tool in clinical [3] and fundamental physiological research [4]. CVR measurements aim at quantifying the cerebral blood flow ( $\mathrm{CBF}$ ) response to changes in circulating gases, most frequently $\mathrm{CO}_{2}$. These measurements have classically been performed using transcranial Doppler ultrasound or positron emission tomography under administration of acetazolamide or in response to a hypercapnia challenge involving breath-hold tasks or respiration of $\mathrm{CO}_{2}$ in air. Functional MRI (fMRI) during a hypercapnia challenge is a recent method to non-invasively map CVR in the entire brain. The study of CVR using fMRI is generally conducted through

\footnotetext{
* Funding by the ARC INRIA AINSI.
} 
the standard BOLD (Blood Oxygen Level Dependent) fMRI modality, due to its relatively high signal-to-noise ratio (SNR). However, the BOLD signal measures perfusion changes indirectly via the venous blood oxygenation and thus depends on several parameters (oxygen consumption, cerebral blood flow and cerebral blood volume), whereas vasoreactivity concerns the arteriolar vasomotor response. As an alternative, a rising fMRI modality is Arterial Spin Labeling (ASL) [5]. ASL has a clear known signal origin in the cerebral blood flow and is more directly related to arteriolar effects. ASL MRI mainly consists of acquiring pairs of control and label images, respectively in the absence and presence of a magnetic labeling of arterial blood upstream of the examined brain tissue, and looking at the average control-label difference. The Signal-to-Noise Ratio (SNR) of this difference is very low so that several tens of image pairs need to be acquired, thus increasing significantly the time spent by the subject in the scanner. Due to this low SNR of ASL, BOLD signals are currently used almost exclusively for CVR measurements, despite the difficulty in the interpretation of this signal if baseline perfusion changes can not be ruled out.

In this work, we analyze ASL data containing both BOLD and perfusion components, acquired during a hypercapnic challenge destined to measure cerebral vasoreactivity (CVR) to $\mathrm{CO}_{2}$. One other reason for the low sensitivity of ASL measurements of CVR may be the fact that it is usually analyzed using a standard linear model (GLM-based) formulation (eg. [6]) with regressors encoding differences in control/tag scans and both ASL and BOLD activation signals being associated with the same canonical response function. The canonical hemodynamic response function (HRF) is often used although it has been been calibrated on BOLD experiments only, thus reflecting simultaneous variations of $\mathrm{CBF}$ and cerebral blood volume (CBV), as well as potential changes in cerebral oxygen consumption $\left(\mathrm{CMRO}_{2}\right)$. In contrast, the perfusion signal only reflects variation in $\mathrm{CBF}$ so that the associated response, that we will call the perfusion response function (PRF) hereafter, is likely to differ from the HRF. The PRF to the hypercapnic stimulus is not well known. Hemodynamic responses in general may moreover be affected by pathology [7]. However, non-linear physiological models of ASL data can address these issues [8]. In the more operational context of linear models, we propose to recover both BOLD response functions (BRF) and perfusion response functions (PRF) from functional ASL data acquired during intermittent inhalation of a small fraction of $\mathrm{CO}_{2}$. We make use of a joint detection estimation (JDE) formalism described in [9]. In the BOLD context, the JDE framework has proven to successfully adapt local HRF estimations while also performing activation detection. This formalism is extended in the current paper to model an additional perfusion component which is embedded in the difference between control and tag scans. Akin to the unknown BRF, a regionspecific unknown PRF is modeled to account for temporal fluctuations across the brain.

After introducing the ASL generative model in Sections 2-3, we give some details on its estimation in Section 4 and perform a comparison with the standard GLM-based approach on a real data set in Section 5 . 


\section{ASL Bayesian hierarchical model}

Following [9], the brain is assumed to have been partitioned into several functional homogeneous parcels, each of which gathers signals which share the same response shapes. The input parcellation is here obtained by spatial clustering of the effect maps estimated by a GLM encoding dispersion around the canonical HRF [10]. Parcels are considered independent, so that the model formulation is hereafter expressed in a single parcel denoted by $\mathcal{P}$. At voxel $j \in \mathcal{P}$ with $|\mathcal{P}|=J$, the ASL time series $\boldsymbol{y}_{j}$ is measured at times $\left(t_{n}\right)_{n=1: N}$ where $t_{n}=n \mathrm{TR}, N$ is the number of scans and TR the time of repetition. The ASL signal is modelled as a linear combination of BOLD and perfusion components, the former remaining the same as the one proposed in [9], while a bilinear and time-invariant system is added to account for the perfusion component. The generative model reads:

$$
\boldsymbol{y}_{j}=c_{j} \boldsymbol{W} \boldsymbol{X} \boldsymbol{g}+a_{j} \boldsymbol{X} \boldsymbol{h}+\boldsymbol{P} \boldsymbol{\ell}_{j}+\alpha_{j} \boldsymbol{w}+\boldsymbol{b}_{j}, \forall j \in \mathcal{P},
$$

where the perfusion and BOLD components are given by the two first terms in Eq. (1). Vectors $\boldsymbol{h} \in \mathbb{R}^{D+1}$ and $\boldsymbol{g} \in \mathbb{R}^{D+1}$ represent the unknown BRF and PRF shapes of size $D+1$ in $\mathcal{P}$, respectively. For the sake of simplicity, the temporal resolution of the BOLD and perfusion response components are the same, without loss of generality. Shapes $\boldsymbol{h}$ and $\boldsymbol{g}$ are constant within parcel $\mathcal{P}$ but the corresponding response levels $a_{j}$ and $c_{j}$, which model the magnitude of activation at voxel $j$, may vary in space across voxels. We denote the BOLD response levels (BRLs) by $\boldsymbol{a}=\left\{a_{j}, j \in \mathcal{P}\right\}$. Similarly, perfusion response levels (PRLs) are denoted by $\boldsymbol{c}=\left\{c_{j}, j \in \mathcal{P}\right\} . \boldsymbol{X}$ denotes the $N \times(D+1)$ binary matrix $\boldsymbol{X}=\left\{x^{n-d \Delta t}, n=1: N, d=0: D\right\}$ that encodes the arrival times of the stimulus occurrences, with $\Delta t<T R$ being the sampling period of the unknown response functions (PRF and BRF). Vector $\boldsymbol{w}$ is $N$-dimensional such that $w_{t_{n}}=1 / 2$ if $t_{n}$ is even (control volume) and $w_{t_{n}}=-1 / 2$ otherwise (tagged volume) and $\boldsymbol{W}=\operatorname{diag}(\boldsymbol{w})$ is the diagonal matrix with $\boldsymbol{w}$ as diagonal components. More precisely, $\boldsymbol{w}$ encodes the difference in magnetization signs between control and tagged ASL volumes. Here we only consider a vasoreactivity protocol involving one single experimental condition but this model can be straightforwardly generalized to encode multiple conditions. Matrix $\boldsymbol{P}=\left[\boldsymbol{p}_{1}, \ldots, \boldsymbol{p}_{O}\right]$ of size $N \times O$ comprises the values at times $t_{n}$ of an orthonormal basis (i.e., $\boldsymbol{P}^{\mathrm{t}} \boldsymbol{P}=\boldsymbol{I}_{O}$ ) consisting of $O$ functions $\boldsymbol{p}_{o}=\left(p_{o, t_{n}}, n=1: N\right)^{\mathrm{t}}$ that take a potential drift and any other nuisance effect (eg. slow motion parameters) into account. Vector $\boldsymbol{\ell}_{j}=\left(\ell_{o, j}, o=1: O\right)^{\mathrm{t}}$ contains the corresponding unknown regression coefficients for voxel $j$. Finally, the scalar $\alpha_{j}$ models the perfusion baseline at voxel $j$. For the sake of simplicity, a white Gaussian noise with unknown variance $v_{b_{j}}$, $\boldsymbol{b}_{j} \sim \mathcal{N}\left(\mathbf{0}, v_{b_{j}} \boldsymbol{I}_{N}\right)$ is considered here but a straightforward extension to $\operatorname{AR}(1)$ noise process can be derived using [9].

\section{Perfusion and hemodynamic priors}

Response shapes. As in [9], the BRF and PRF shapes are assumed to follow prior multivariate Gaussian distributions that respectively read $\boldsymbol{h} \sim \mathcal{N}\left(\mathbf{0}, v_{\boldsymbol{h}} \boldsymbol{\Sigma}\right)$ 
and $\boldsymbol{g} \sim \mathcal{N}\left(\mathbf{0}, v_{\boldsymbol{g}} \boldsymbol{\Sigma}\right)$, where $v_{\boldsymbol{h}}$ and $v_{\boldsymbol{g}}$ are unknown variances and $\boldsymbol{\Sigma}$ a fixed covariance matrix encoding a constraint on the second order derivative so as to account for temporal smoothness. It follows different and unconstrained shapes for the PRF and BRF.

Response Levels. The BOLD (BRLs) and perfusion (PRLs) response levels are assumed to follow spatial Gaussian mixture models with different means and variances but governed by a common hidden Markov random field encoding voxels activation states. To encode the activation states, class assignment variables $\boldsymbol{q}=\left\{q_{j}, j \in \mathcal{P}\right\}$ are introduced where $q_{j}$ is the activation class at voxel $j$. Two classes are considered, for activated $(i=2)$ and non-activated $(i=1)$ voxels. Formally, BRLs and PRLs are a priori independent conditionally to $\boldsymbol{q}$ :

$$
p(\boldsymbol{a}, \boldsymbol{c} \mid \boldsymbol{q})=\prod_{i=1}^{2} \prod_{j \in J_{i}}\left[\mathcal{N}\left(a_{j} ; \mu_{i}, v_{i}\right) \mathcal{N}\left(c_{j} ; \eta_{i}, \rho_{i}\right)\right],
$$

with $J_{i}=\left\{j \in J \mid q_{j}=i\right\}$ and $\mu_{i}, v_{i}$ and $\eta_{i}, \rho_{i}$ are the unknown means and variances of the $i^{\text {th }}$ mixture component, for the BRLs and PRLs, respectively. As in [9], spatial correlation is introduced through an Ising model on $\boldsymbol{q}$ :

$$
p(\boldsymbol{q} \mid \beta)=Z(\beta)^{-1} \exp \left(\beta \sum_{j \sim j^{\prime}} \mathbb{1}\left(q_{j}=q_{j^{\prime}}\right)\right)
$$

where $j \sim j^{\prime}$ indicates that the sum extends over all neighboring pairs of voxels. We denote $\mathbb{1}(A)=1$ if $A$ is true and $\mathbb{1}(A)=0$ otherwise. $\beta$ is the spatial regularization factor and $Z(\beta)$ is the partition function.

Perfusion baseline. A priori, this quantity should not be difficult to extract as it roughly corresponds to the mean over the differences between control and tagged volumes. Hence, a simple Gaussian prior is introduced: $\forall j, \alpha_{j} \sim \mathcal{N}\left(0, v_{\boldsymbol{\alpha}}\right)$ and $\boldsymbol{\alpha}=\left\{\alpha_{j}, j \in \mathcal{P}\right\}$.

Drift coefficients. The prior on these coefficients $\boldsymbol{L}=\left\{\boldsymbol{\ell}_{j}, j \in \mathcal{P}\right\}$ is Gaussian: $\forall j, \ell_{j} \sim \mathcal{N}\left(\mathbf{0}, v_{\ell} \boldsymbol{I}_{O}\right)$.

Hyper-parameters. Non-informative Jeffreys priors are adopted for $\left\{v_{\boldsymbol{b}}, v_{\boldsymbol{\ell}}, v_{\boldsymbol{\alpha}}\right.$, $\left.v_{\boldsymbol{g}}, v_{\boldsymbol{h}}\right\}$ and proper conjugate priors are considered for the mixture parameters of BRLs and PRLs $\boldsymbol{\theta}_{\boldsymbol{a}}=\left\{\mu_{i}, v_{i}, i=1: 2\right\}$ and $\boldsymbol{\theta}_{\boldsymbol{c}}=\left\{\eta_{i}, \rho_{i}, i=1: 2\right\}$. The positive spatial correlation parameters $\beta$ is associated with a uniform prior over $[0,1.5]$ as values over 1.5 correspond to completely correlated fields [9]. All hyper-parameters to estimate are grouped in the set $\boldsymbol{\Theta}$.

\section{Bayesian MCMC Inference}

To sample the posterior of interest $p(\boldsymbol{a}, \boldsymbol{c}, \boldsymbol{q}, \boldsymbol{h}, \boldsymbol{g}, \boldsymbol{L}, \boldsymbol{\alpha}, \boldsymbol{\Theta} \mid \boldsymbol{y})$, each variable $x \in \mathcal{X}$ $(\mathcal{X}=\{\boldsymbol{a}, \boldsymbol{c}, \boldsymbol{q}, \boldsymbol{h}, \boldsymbol{g}, \boldsymbol{L}, \boldsymbol{\alpha}, \boldsymbol{\Theta}\})$ is drawn in turn conditionally to $\boldsymbol{y}$ and the other variables in $\mathcal{X} \backslash x$, using a hybrid Metropolis-Gibbs sampling scheme and posterior mean estimates (PM) are computed from the samples after a burn-in period (PM estimators ensure the minimization of the mean square error and are the most 
widely used in the Bayesian framework). The sampling convergence was tracked by a criterion on the successive PM estimates. The computation time of the ASL-JDE approach is around 3 times larger than the BOLD only version [9] and amounts to $\sim 20$ hours on a $2.4 \mathrm{GHz} \mathrm{CPU}$ for a whole brain analysis.

Posterior conditional distributions $p\left(x^{k} \mid(\mathcal{X} \backslash x)^{k-1}, \boldsymbol{y}\right)$ computed at each iteration $k$ of the sampling scheme are identifiable to those derived in [9], except for the ones which are detailed hereafter. Iteration index $k$ is dropped hereafter. Assignment variables. The full conditional posterior of $\boldsymbol{q}$ is identified to an asymmetric Ising field:

$$
\begin{aligned}
& p\left(q_{j} \mid \mathcal{X} \backslash q_{j}\right) \propto \exp \left[\beta U\left(\kappa_{j}\right)+\sum_{i=1}^{2} \mathbb{1}\left(q_{j}=i\right) S_{i, j}\right] \\
& \text { with: } U\left(\kappa_{j}\right)=\sum_{j^{\prime} \in \kappa_{j}} \mathbb{1}\left(q_{j}=q_{j^{\prime}}\right), \text { where } \kappa_{j}=\{\text { neighbors of } j\}, \text { and } \\
& S_{i, j}=\left(-\frac{\ln \left(v_{i} \rho_{i}\right)}{2}-\frac{\left(a_{j}-\mu_{i}\right)^{2}}{2 v_{i}}-\frac{\left(c_{j}-\eta_{i}\right)^{2}}{2 \rho_{i}}\right) .
\end{aligned}
$$

BRLs and PRLs. Let us introduce the following notation for partial residual quantities: $\overline{\boldsymbol{y}}_{j}=\boldsymbol{y}_{j}-P \boldsymbol{\ell}_{j}-\alpha_{j} \boldsymbol{w}, \widehat{\boldsymbol{y}}_{j}=\overline{\boldsymbol{y}}_{j}-c_{j} \boldsymbol{W} \boldsymbol{X} \boldsymbol{g}, \breve{\boldsymbol{y}}_{j}=\overline{\boldsymbol{y}}_{j}-a_{j} \boldsymbol{X} \boldsymbol{h}$. The BRLs and PRLs follow the full conditional posterior distributions given by:

$$
\begin{array}{r}
\left(a_{j} \mid q_{j}=i, \ldots\right) \sim \mathcal{N}\left(\frac{\boldsymbol{\epsilon}^{T} \widehat{\boldsymbol{y}}_{j}+v_{b_{j}} \mu_{i}}{\left(v_{i, j}\right)^{-1} v_{b_{j}} v_{i}}, v_{i, j}\right), \\
\text { with } \boldsymbol{\epsilon}=(\boldsymbol{X})^{T} \boldsymbol{h} \quad \text { and } \quad v_{i, j}=\frac{v_{b_{j}} v_{i}}{\boldsymbol{\epsilon}^{T} \boldsymbol{\epsilon} v_{i}+v_{b_{j}}}, \\
\left(c_{j} \mid q_{j}=i, \ldots\right) \sim \mathcal{N}\left(\frac{\boldsymbol{\zeta}^{T} \breve{\boldsymbol{y}}_{j}+v_{b_{j}} \eta_{i}}{\left(\rho_{i, j}\right)^{-1} v_{b_{j}} \rho_{i}}, \rho_{i, j}\right), \\
\text { with } \boldsymbol{\zeta}=\boldsymbol{W}(\boldsymbol{X})^{T} \boldsymbol{g} \quad \text { and } \quad \rho_{i, j}=\frac{v_{b_{j}} \rho_{i}}{\boldsymbol{\zeta}^{T} \boldsymbol{\zeta} \rho_{i}+v_{b_{j}}}
\end{array}
$$

Perfusion baseline. The partial residual quantity involved here is denoted: $\widetilde{\boldsymbol{y}}_{j}=\boldsymbol{y}_{j}-a_{j} \boldsymbol{X} \boldsymbol{h}-c_{j} \boldsymbol{W} \boldsymbol{X} \boldsymbol{g}-P \boldsymbol{\ell}_{j}-\alpha_{j} \boldsymbol{w}$. The full conditional posterior distribution of the perfusion baseline reads:

$$
\left(\alpha_{j} \mid \mathcal{X} \backslash \alpha_{j}, \boldsymbol{y}_{j}\right) \sim \mathcal{N}\left(\frac{v_{\boldsymbol{\alpha}} \boldsymbol{W}^{T} \widetilde{\boldsymbol{y}}_{j}}{N v_{\boldsymbol{\alpha}}+v_{b_{j}}}, \frac{v_{\boldsymbol{\alpha}} v_{b_{j}}}{N v_{\boldsymbol{\alpha}}+v_{b_{j}}}\right)
$$

\section{Results}

Real data were acquired on a healthy subject with 3-T MRI (Philips Achieva TX scanner), using a 32-channel head-only receive array and a pseudo-continuous ASL (pCASL) sequence [11] during a block-design inhalation paradigm to measure CVR to $\mathrm{CO}_{2}$ and basal CBF maps. PCASL acquisition parameters were: WET pre-saturation, $1650 \mathrm{~ms}$ label, $1525 \mathrm{~ms}$ post-label delay, multi-slice singleshot EPI readout $\left(3 \times 3 \times 6 \mathrm{~mm}^{3}, 20\right.$ slices, TE $12 \mathrm{~ms}$, sense-factor 2.5$)$, TR of 
$4 \mathrm{~s}$. A total of 180 control and tag images were acquired in 12 min. Capnia was modulated during the pCASL acquisition in a 1/2/1-min paradigm (3 cycles) by alternating medical air and an air $/ \mathrm{CO}_{2}$ mixture $\left(7 \% \mathrm{CO}_{2}, 21 \% \mathrm{O}_{2}\right.$, balance $\left.\mathrm{N}_{2}\right)$ administered at $121 . \mathrm{min}^{-1}$. Data were pre-processed using the SPM8 software and custom routines.

The pre-processed images were then analyzed using either the-based JDE approach previously described or a GLM approach as implemented in SPM8. The latter consisted of a design matrix comprising four regressors as in [6]: i.) a perfusion baseline (alternating sequence of $-1 / 2,1 / 2$ ), ii.) a BOLD component obtained from the convolution of the block paradigm with the canonical HRF, iii.) a vasoreactive perfusion component obtained by multiplying regressors i.) and ii.), and iv.) a unit baseline regressor. Low frequency drifts were estimated by a least-square fit of polynomial functions. Note that for all results, the scale of signals is in arbitrary units.

(a)

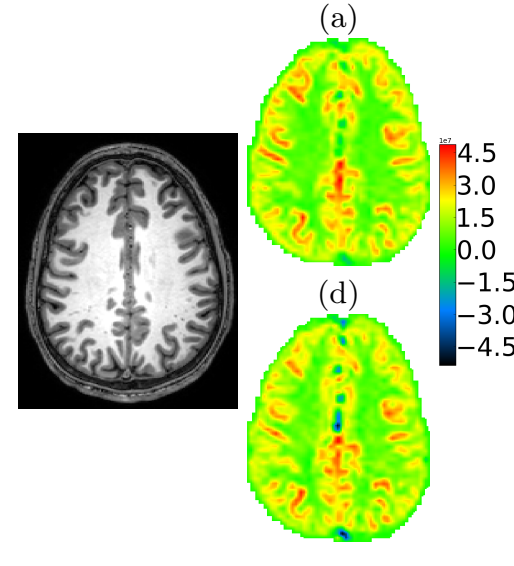

(b)

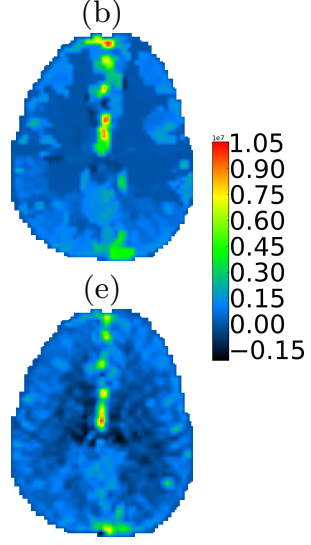

(c)

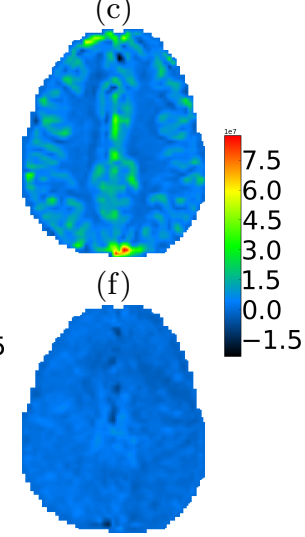

Fig. 1. Comparison of perfusion and BOLD maps on real data for the JDE (top row) and GLM (bottom row) analyses. (a,d): Basal perfusion levels estimated by JDE $(\widehat{\boldsymbol{\alpha}})$ and GLM, respectively. $(\mathbf{b}, \mathbf{e})$ : Vaso-reactive perfusion levels estimated by JDE $(\hat{\boldsymbol{c}})$ and GLM, respectively. (c,f): BOLD levels estimated by JDE $(\widehat{\boldsymbol{a}})$ and GLM, respectively. The corresponding anatomical slice is depicted on the left part.

For the perfusion baseline estimates, the JDE-based approach (Fig. 1(a)) yields slightly more uniform values than the GLM approach (Fig. 1(d)) whose values in the anterior part of the brain are lower than those in posterior part. Indeed, we expect homogeneous CBF values across the whole brain. The contrast between gray and white matter is also more enhanced with JDE than with GLM, as confirmed in Fig. 2(a,b) where the distributions of perfusion baseline levels between gray and white matter are more segregated by JDE. This higher contrast between gray and white matter with JDE is also observed for the vasoreactive perfusion levels (compare Fig. 1(b,e) and Fig. 2(c,d)). Levels in the 
white matter levels are especially more regularized using JDE. Maps of BOLD levels in Fig. 1(c,f) show higher sensitivity for JDE which enables a better fit of the BOLD component as confirmed in Fig. 3. In this figure, the fitted block BOLD response is especially more well fitted in the transition parts with JDE. The overall goodness-of-fit is also better with JDE.

(a)

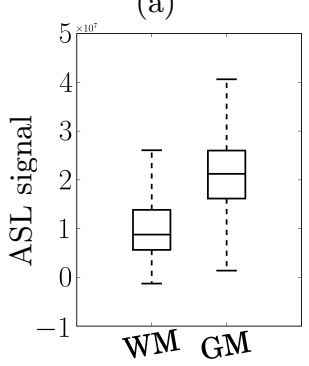

(b)



(c)

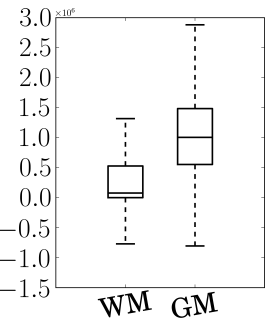

(d)

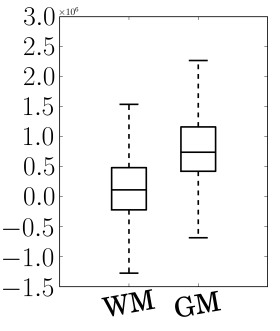

Fig. 2. Box plots of perfusion levels within gray (GM) and white (WM) matter, for the JDE $(\mathbf{a}, \mathbf{c})$ and GLM $(\mathbf{b}, \mathbf{d})$ approaches. $(\mathbf{a}, \mathbf{b})$ : distributions of perfusion baseline levels. $(\mathbf{c}, \mathbf{d})$ : distributions of vaso-reactive perfusion levels.
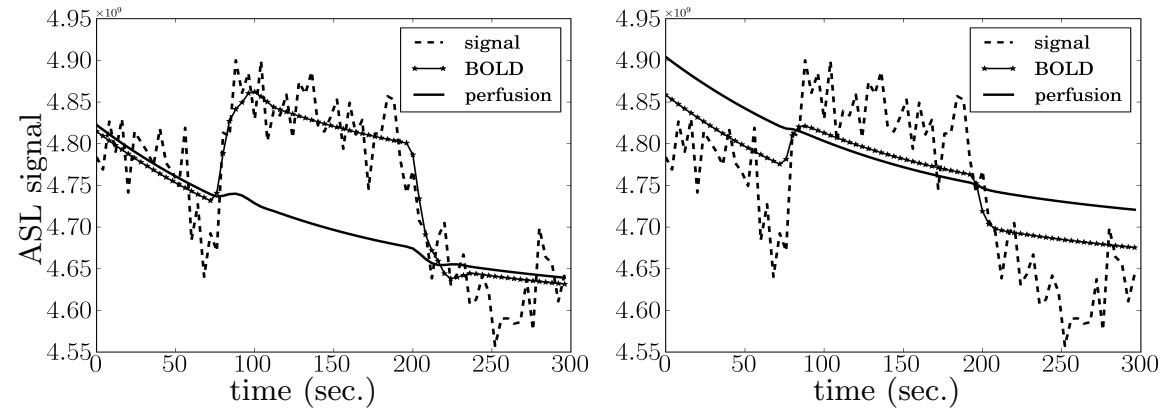

Fig. 3. Comparison of signal fitting for the JDE (left) and GLM (right) approaches, for one block of hypercapnia (the remaining two are not shown). The perfusion components have been multiplied by $\boldsymbol{W}$ to clearly show their variations, and their baseline is here arbitrary since they are encoded in the difference of two subsequent scans.

\section{Conclusion}

The Bayesian hierarchical approach presented here is able to perform BOLD and perfusion source separation and achieves better than the standard GLM-based 
analysis in terms of goodness-of-fit and also sensitivity, especially in its ability to better segregate the gray and white matter within the estimated perfusion levels. Future work will focus on generalizing our study to a group of subjects and provide more quantitative results. Methodological extensions will improve the modeling of the perfusion response function with a positivity constraint. A hierarchical prior modeling is also envisaged to couple the responses in the BOLD and perfusion components. Finally, as the input parcellation has only been validated in the BOLD-only context [12], it will be further tested in the ASL context.

\section{References}

1. Krainik, A., Hund-Georgiadis, M., Zysset, S., von Cramon, D.Y.: Regional impairment of cerebrovascular reactivity and BOLD signal in adults after stroke. Stroke 36 (2005) 1146-52

2. Cantin, S., Villien, M., Moreaud, O., Tropres, I., Keignart, S., Chipon, E., Le Bas, J.F., Warnking, J., Krainik, A.: Impaired cerebral vasoreactivity to $\mathrm{CO} 2$ in Alzheimer's disease using BOLD fMRI. Neuroimage 58 (2011) 579-87

3. Attyé, A., Villien, M., Tahon, F., Warnking, J., Detante, O., Krainik, A.: Normalization of cerebral vasoreactivity using BOLD MRI after intravascular stenting. Hum Brain Mapp (2013)

4. Villien, M., Bouzat, P., Rupp, T., Robach, P., Lamalle, L., Troprès, I., Estève, F., Krainik, A., Lévy, P., Warnking, J., Verges, S.: Changes in cerebral blood flow and vasoreactivity to $\mathrm{CO}(2)$ measured by Arterial Spin Labeling after 6 days at 4,350 m. Neuroimage (2013)

5. Liu, T.T., Brown, G.: Measurement of cerebral perfusion with arterial spin labeling: Part 1. methods. Journal of the International Neuropsychological Society 13 (2007) $517-525$

6. Hernandez-Garcia, L., Jahanian, H., Rowe, D.B.: Quantitative analysis of arterial spin labeling FMRI data using a general linear model. Magnetic Resonance Imaging 28 (2010) 919-927

7. Roc, A., Wang, J., Ances, B., Liebeskind, D., Kasner, S., Detre, J.: Altered hemodynamics and regional cerebral blood flow in patients with hemodynamically significant stenoses. Stroke 37 (2006) 382-7

8. Woolrich, M., Chiarelli, P., Gallichan, D., Perthen, J., Liu, T.: Bayesian inference of hemodynamic changes in functional arterial spin labeling data. Magn Reson Med. 56 (2006) 891-906

9. Vincent, T., Risser, L., Ciuciu, P.: Spatially adaptive mixture modeling for analysis of within-subject fMRI time series. IEEE Trans. Med. Imag. 29 (2010) 1059-1074

10. Thirion, B., Flandin, G., Pinel, P., Roche, A., Ciuciu, P., Poline, P.: Dealing with the shortcomings of spatial normalization: Multi-subject parcellation of fMRI datasets. Hum. Brain Mapp. 27 (2006) 678-693

11. Dai, W., Garcia, D., de Bazelaire, C., Alsop, D.C.: Continuous flow-driven inversion for arterial spin labeling using pulsed radio frequency and gradient fields. Magn Reson Med 60 (2008) 1488-97

12. Vincent, T., Ciuciu, P., Thirion, B.: Sensitivity analysis of parcellation in the joint detection-estimation of brain activity in fMRI. In: 5th Proc. IEEE ISBI, Paris, France (2008) 568-571 